\title{
A Model Parameter Extraction Method for Dielectric Barrier Discharge Ozone Chamber using Differential Evolution
}

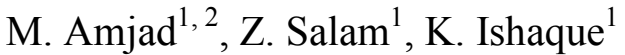 \\ ${ }^{1}$ Center of Electrical Energy Systems, Faculty of Electrical Engineering, Universiti Teknologi Malaysia, 81310, Johor, \\ Malaysia, \\ ${ }^{2}$ University College of Engineering and Technology, The Islamia University of Bahawalpur, 63100, Punjab, Pakistan. \\ Muhammad.Amjad@iub.edu.pk, zainals@fke.utm.my
}

\begin{abstract}
In order to design an efficient resonant power supply for ozone gas generator, it is necessary to accurately determine the parameters of the ozone chamber. In the conventional method, the information from Lissajous plot is used to estimate the values of these parameters. However, the experimental setup for this purpose can only predict the parameters at one operating frequency and there is no guarantee that it results in the highest ozone gas yield. This paper proposes a new approach to determine the parameters using a search and optimization technique known as Differential Evolution (DE). The desired objective function of DE is set at the resonance condition and the chamber parameter values can be searched regardless of experimental constraints. The chamber parameters obtained from the DE technique are validated by experiment.
\end{abstract}

Keywords: Dielectric barrier discharge, differential evolution, Lissajous figure, ozone chamber, parameter estimation.

\section{INTRODUCTION}

$\mathrm{I}^{\mathrm{N}}$ RECENT YEARS, the use of ozone gas $\left(\mathrm{O}_{3}\right)$ as a disinfecting and oxidizing agent has been steadily increasing. It is widely used in industrial, domestic, agriculture, food processing, and pharmaceutical applications. In industry, ozone is used for bleaching pipelines and waste water treatment. Increasingly, it has been used as an alternative to chlorine for water purification. In agriculture applications, ozone-enriched water is used for hydroponics and for the removal of pesticide residue from fruits and vegetables. Ozone-enriched air is used in cold storages to increase the shelf life of vegetables and fruits [1]. Many hospitals have been using ozone to disinfect laundries and to sanitize critical equipment. The one most important advantage of ozone over other oxidizing agents is that it leaves no residues harmful to global environment

The most economical method to generate ozone is by utilizing the electrical discharge at atmospheric pressure. It is done by applying high voltage to an ozone chamber filled with air or oxygen. The chamber consists of two electrodes, in which at least one of them is covered with dielectric. The conventional and most commonly used material for dielectric is glass. An adequate spacing between electrodes (known as discharge gap) is introduced to allow for the air or oxygen to pass through. The electrical discharge created in this type of chamber is known as Dielectric Barrier Discharge (DBD) [2-3]. The DBD chamber is considered as a source of non-thermal plasma which operates at atmospheric pressure. When the magnitude of AC voltage applied to the electrodes is greater than the initiation voltage, the energy in the discharge gap breaks the oxygen $\left(\mathrm{O}_{2}\right)$ molecules into single $\mathrm{O}$ atom. The latter combines with $\mathrm{O}_{2}$ to form ozone.

Traditionally, the DBD chamber utilizes low frequency $(50 \mathrm{~Hz})$, high voltage AC. However, with the advent of power electronics technologies, the use of high frequency resonant power supplies is increasing. The advantage of high frequency is the increased power density applied to the chamber electrode surface, resulting in higher ozone production. Alternatively, the same amount of ozone can be achieved even if the applied peak voltage to the chamber is decreased (but must be above the initiation voltage). This is done by increasing the frequency of the AC power supply to the chamber. The lower voltage allows for the use of nonconventional dielectric material such as mica which is much cheaper and easier to fabricate [4].

To design an efficient high-voltage, high-frequency power supply, it is necessary to know the parameters of the chamber. Various electrical models for the chamber are proposed; in [5-6] a non-linear electrical model of ozone chamber is presented. This model has three parameters, namely capacitance due to discharge gap $\left(C_{a}\right)$, capacitance of the dielectric sheet $\left(C_{g}\right)$ and discharge initiating voltage of the chamber $\left(V_{z}\right)$. The main disadvantage of the model is that, it does not account for the losses in the electrodes. Later, a simplified version of this model (linear model) that includes electrode losses is proposed [7]. This model involves two parameters, namely chamber capacitance $\left(C_{p}\right)$ and chamber resistance $\left(R_{p}\right)$. The power consumed by the chamber in the form of heat is represented by power dissipated in the equivalent resistance $R_{p}$. In another work [8-9], an improved model considering the electrode losses in the non-linear model [5] is presented. Regardless of the type of chamber used, it is necessary to match the impedance of chamber with the resonant circuit of the power supply for maximum power transfer. Therefore, the accurate estimation of chamber parameters is important for designing an efficient ozone power supply.

Typically, the parameter values are calculated by the information extracted from the Lissajous plot. However, chamber parameter estimation using this technique has several disadvantages. First, it cannot provide the parameter values over a range of frequencies. The experimental setup, which consists of RF amplifier, high-turn ratio transformer, 
and capacitor can only predict the parameters at one operating frequency [7]. Typically this frequency is near the resonance to ensure maximum voltage is fed to the chamber. However, there is no guarantee that the applied frequency results in the highest ozone gas yield [10]. This is due to the fact that the chamber parameters vary unpredictably (non-linear) with frequency, thus altering its resonance. Hence, it is important for the designer to know the parameter values over a wide range of frequency so that the power supply design can be optimized. Second, experimental results show that the shape of the Lissajous plot is frequency dependent. At frequencies around $10 \mathrm{kHz}$, the shape is parallelogram-like. Using this shape, both linear and non-linear chamber parameters can be accurately estimated. However, in the frequency range of 15$20 \mathrm{kHz}$, the figure changes to circular; with this shape only linear model parameters can be estimated. At higher frequency (above $20 \mathrm{kHz}$ ), the Lissajous figure becomes distorted; parameter estimation becomes extremely inaccurate [11].

Whilst it is preferable to obtain the chamber parameters analytically, to the best knowledge of the author, no previous attempt was made along this approach. Although the voltages gain at resonance can be derived, there exist three unknown variables with only two governing equations. In these circumstances, an analytical approach may not give the best solution because one of the parameter values has to be preassumed. The problem is, if the pre-assumption value is incorrect, the computation for the other two values is erroneous. The other option is to use computational optimization techniques such as numerical iteration and direct search. The latter, generally known as the Evolutionary Algorithm (EA), is interesting because a desired objective function can be set at the resonance condition and the chamber parameter values can be searched regardless of experimental constraints.

EA is a stochastic optimization method that appears to be very efficient in optimizing real-valued multi-modal objective functions. Various EA techniques such as genetic algorithm (GA), particle swarm optimization (PSO) and Differential evolution (DE) are used for parameter estimation in many other areas [12-13-14]. However, DE stands out due to its structural simplicity and robustness. Furthermore, it utilizes fewer control parameters while yielding good accuracy. Considering these advantages, this paper proposes a simple and efficient method to extract the parameters of ozone chamber using the DE. Unlike other methods, the chamber parameters can be estimated simultaneously together with the operating frequency, i.e. it will yield the best combinations of $C_{p}$ and $R_{p}$ at a specific resonance condition. The proposed method, therefore, offers an improved alternative to the conventional Lissajous plots.

The remaining paper is organized as follows: Section 2 describes the details of the circuit model for ozone chamber. Section 3 explains the background of the DE theory. Section 4 describes how this technique is used to extract the model parameters. Chamber parameter extracted results are given in Section 5. Validation of the proposed method is given in Section 6. Section 7 draws the conclusions of this paper.

\section{Circuit MOdel OF OZONE CHAMBER}

\section{A. Non-linear model.}

In Fig.1.a), the non-linear electrical model of ozone chamber is shown [5]. It consists of diodes, DC voltage source $\left(V_{z}\right)$, and capacitors $C_{a}$ and $C_{g}$. The voltage source represents the voltage at which the DBD is initiated. Capacitors $C_{a}$ and $C_{g}$ represent the capacitances due to discharge gap and dielectric sheet, respectively. The voltage $V_{z}$ is estimated by measuring the distance from centre to cross point of voltage-charge $(V-Q)$ Lissajous plot as shown in Fig.1.b). The values of capacitors $C_{g}$ and $C_{a}$ were estimated as follows:

$$
\begin{gathered}
\tan \phi_{1}=C_{g} \cdot S \\
\tan \phi_{2}=\frac{C_{a} \cdot C_{g} \cdot S}{C_{a}+C_{g}}
\end{gathered}
$$

where $S$ is the area of chamber electrode and the inclinations $\Phi_{1}, \Phi_{2}$ are obtained from $V$ - $Q$ Lissajous plot.

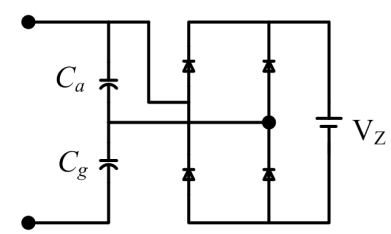

(a)

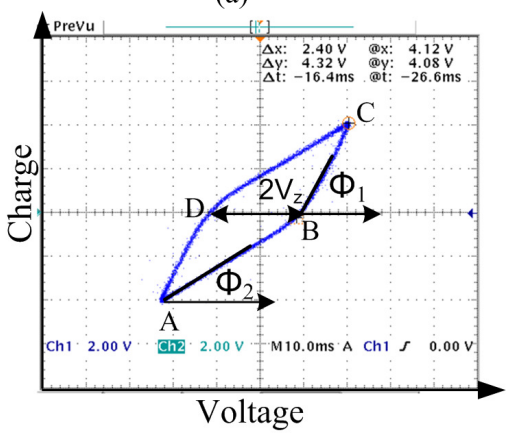

(b)

Fig.1. a) Non-linear model of ozone chamber b) Voltage-charge Lissajous plot.

\section{B. Linear model.}

Fig.2.a) shows the linear model of ozone chamber as proposed in [7]. In this model, the ozone chamber is represented by the parallel combination of resistor $\left(R_{p}\right)$ and capacitor $\left(C_{p}\right)$. The chamber model parameters are obtained based on voltage-charge $(V-Q)$ and voltage-current $(V-I)$ Lissajous plots. The values of $C_{p}$ and $R_{p}$ are calculated using (3) and (4), i.e.

$$
C_{p}=\frac{I_{o}}{2 \pi f V_{m}}
$$




$$
R_{p}=\frac{V_{m}}{2 \pi f Q_{0}}
$$

where $V_{m}$ is the peak value of sinusoidal voltage of chamber and $f$ is the frequency of the applied signal to the chamber. The values of $I_{o}, Q_{o}$ are obtained from $V-I$ and $V-Q$ Lissajous plots, as shown in Fig.2.b) and 2.c), respectively.

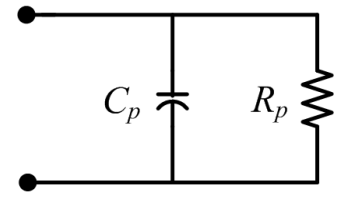

(a)

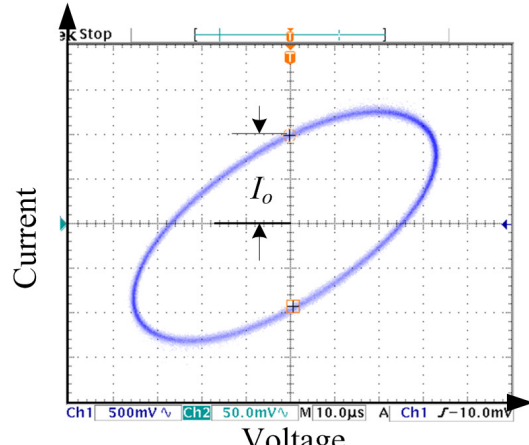

(b)

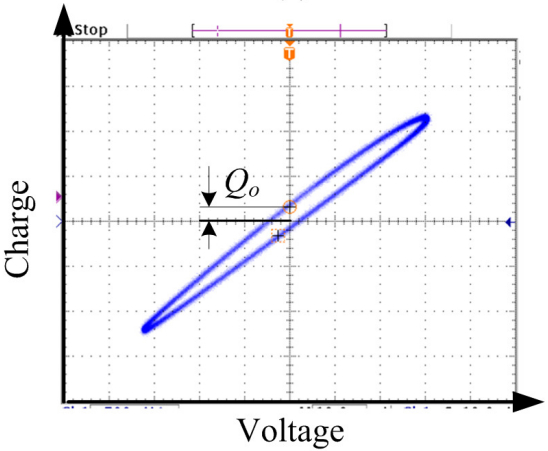

(c)

Fig.2. a) Linear model of ozone chamber. Experimental measurement of Lissajous plot b) voltage-current c) voltagecharge.

\section{DifFERENTIAL EVOLUTION (DE)}

$\mathrm{DE}$ is an optimization procedure, analogous to the genetic algorithm (GA). In contrast to GA, which relies on crossover, DE mainly employs mutation operation (i.e. difference vector) as the exploration and selection mechanism to direct the search toward the prospective regions in the search space. Like other members in the EA family, DE initiates with a random population generation, which is then improved using selection, mutation, and crossover. The process is iterated through generations until the stopping condition is reached; usually a reasonably good fitness value or a predefined maximum number of generations $G_{\max }$.

Similar to the other EA, DE also works on a population, $P_{G}$, of the candidate solutions. These candidate solutions are known as the individuals of the population. In particular, DE creates a population of $N P \times D$ dimensional real-valued parameter vectors $X_{i, G}$ as:

$$
\begin{gathered}
P_{X, G}=\left(X_{i, G}\right) i=1,2, \ldots \ldots, N P, G=0,1, \ldots \ldots G_{\max } \\
X_{i, G}=\left(X_{j, i, G}\right) j=1,2, \ldots \ldots ., D
\end{gathered}
$$

The index, $G=0,1, \ldots, G_{\max }$, indicates the generation to which a vector belongs. In addition, each vector has a population index, $i$, from 1 to $N p$. The size of population is adjusted by $N p$. Parameters within vectors are indexed with $j$, from 1 to $D$. The DE process involves the following steps:

\section{A. Initialization.}

To begin the optimization process, an initial population of $N P \times D$ dimensional real-valued parameter vectors $X_{i, G}=\left[X_{1, i, G}, X_{2, i, G}, \ldots . ., X_{j, i, G}, \ldots \ldots, X_{D, i, G}\right] \quad$ is created. The population is randomly initialized from initial parameter bounds. Each vector forms a candidate solution to the multidimensional optimization problem. Initial parameter values are usually randomly selected uniformly in the interval $\left[X_{L}, X_{H}\right]$. The $X_{L}=\left[X_{1, L}, X_{2, L}, \ldots \ldots X_{D, L}\right]$ and $X_{H}=$ $\left[X_{1, H}, X_{2, H}, \ldots \ldots, X_{D, H}\right]$ are the lower and upper bound of the search space, respectively.

$$
X_{j, i, 0}=X_{L}+\operatorname{rand}[0,1]\left(X_{H}-X_{L}\right)
$$

where $\operatorname{rand}[0,1]$ is a random number uniformly distributed between 0 and 1 . The initialization procedure of $\mathrm{DE}$ is demonstrated in Fig.3.a).

\section{B. Mutation}

Mutation is a perturbation with a random element. A predefined probability density function is not used by DE to generate perturbing fluctuations. DE relies upon the population itself to perturb the vector parameter. In the nomenclature of $\mathrm{DE}$, a parent vector from the current generation is known as target vector, a mutant vector achieved through the differential mutation operation is called a donor vector, and lastly an offspring formed by recombining the donor with the target vector is called the trial vector. For a given parameter vector $X_{i, G}$ three distinct random vectors

$\left(X_{r l, G}, X_{r 2, G}, X_{r 3, G}\right)$ are selected in the range $[1, N P]$.

Then, a donor vector $V_{i, G}$ is created by adding the weighted difference between the two vectors to the third (base) vector as:

$$
V_{i, G}=X_{r 1, G}+F\left(X_{r 2, G}-X_{r 3, G}\right)
$$

where $F$ is a mutation scaling factor and it is typically chosen from the range $[0,1]$. It is used to control the differential variation $d_{i}=\left(X_{r 2, G}-X_{r 3, G}\right)$. Fig.3.b) demon- 
strates the process on a 2-D parameter space, which shows the constant cost contours of an arbitrary fitness function.

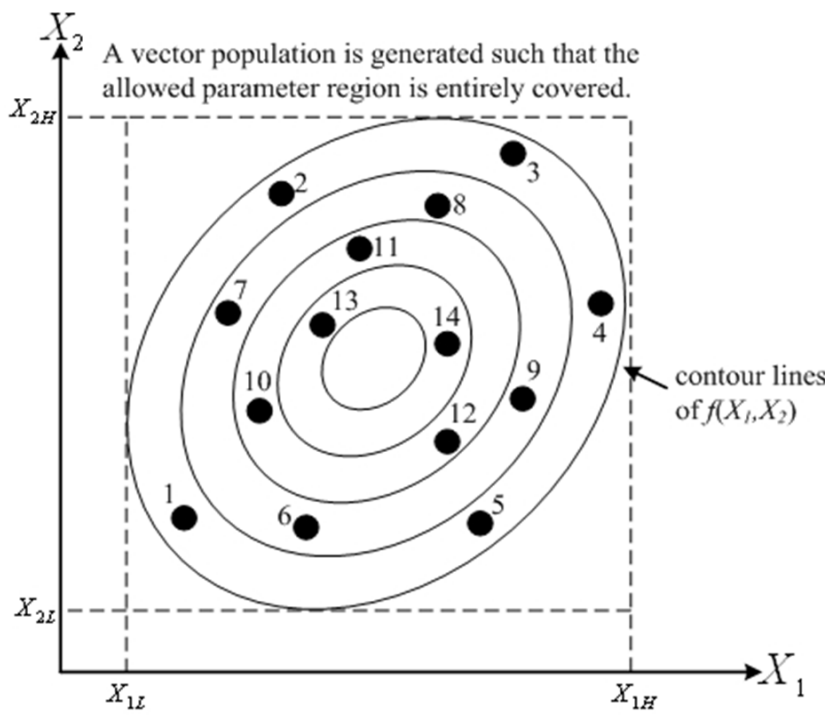

(a)

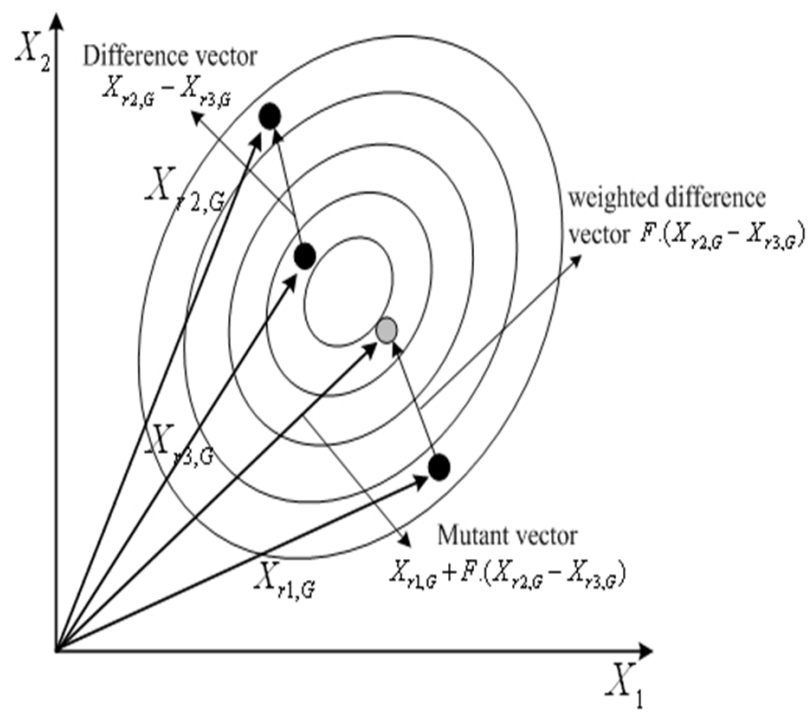

(b)

Fig.3. a) Initialization of DE b) Mutation process of DE [16].

\section{Crossover.}

The donor vector $V_{i, G+1}$ and the target vector $X_{i, G}$ are mixed to yield the trial vector. It can be represented by the following equation:

$$
U_{i, G}=\left[U_{1 i, G}, U_{2 i, G}, \ldots . . U_{j i, G}, \ldots ., U_{D i, G}\right]
$$

In DE algorithm, two kinds of crossover methods are used, i.e. binomial and exponential [15]. In this work, exponential crossover strategy is used which can be described by the following pseudo-code:

$$
\begin{aligned}
& U_{i, G}=X_{i, G} ; \\
& \mathrm{k}=\mathrm{i}(\operatorname{rand}\{1, \ldots, \mathrm{n}\}) ; \\
& j=k ; \\
& L=0 \\
& \text { WHILE rand }[0,1]<C R A N D L<N P \\
& U_{i, G j}=V_{i, G, \mathrm{j}} ; \\
& j=j+1 ; \\
& L=L+1 \\
& \text { END WHILE }
\end{aligned}
$$

where, $C R$ is known as the crossover rate and appears as another control parameter of DE just like $F . j \in[1,2, \ldots \ldots . D]$ is a randomly chosen index, which ensures that $U_{i, G}$ attains at least one element from $V_{i, G}$.

To ensure the solution remains in feasible region, a penalty function is used [16]. It authenticates that the parameter values always lie within the allowable range after recombination. Any parameter that violates the limits is replaced with the following penalty function:

$U_{i, G+1}=\left\{\begin{array}{l}U_{i, G+1}-\operatorname{rand}[0,1]\left(X_{i H}-X_{i L}\right) i f U_{i, G+1}>X_{i H} \\ U_{i, G+1}+\operatorname{rand}[0,1]\left(X_{i H}-X_{i L}\right) i f U_{i, G+1}>X_{i L}\end{array}\right.$

\section{Evaluation and selection.}

The selection operation at $G=G+1$ is described by the following equation:

$$
X_{i, G+1}= \begin{cases}U_{i, G} & \text { if } J\left(U_{i, G}\right)<J\left(X_{i, G}\right) \\ X_{i, G} & \text { otherwise }\end{cases}
$$

where, $J(X)$ is the objective function to be minimized, while $i \in\left[1, N_{p}\right]$. As a result, if the new trial vector attains a lower value of the objective function, it exchanges the corresponding target vector in the next generation; otherwise the target is preserved in the current population. Hence, the population either acquires a better or remains the same in fitness status, but never declines.

\section{Proposed MOdelling METHOD USING DE}

The linear chamber model considered in this work is characterized as highly capacitive loads [17]. The variable inductor $\left(L_{s}\right)$ is connected in series with chamber, making it a parallel-loaded resonant topology as shown in Fig.4. If the input signal frequency is at resonance, the voltage and current waveform of the chamber are expected to be pure sinusoidal. The input impendence and transfer function of this circuit is given by:

$$
Z_{\text {in }}=\frac{R_{P}}{1+\left(\omega R_{P} C_{P}\right)^{2}}+j\left(\omega L_{s}-\frac{\omega R_{g}{ }^{2} C_{P}}{1+\omega R_{P}{ }^{2} C_{P}^{2}}\right)
$$




$$
A_{v}=\left|\frac{V_{o}(j \omega)}{V_{i n}(j \omega)}\right|=\frac{1}{\sqrt{\left(1-\left(\frac{\omega}{\omega_{p}}\right)^{2}\right)^{2}+\left(\frac{\omega}{\omega_{p} Q_{p}}\right)^{2}}}
$$

where $\omega_{p}$ is undamped natural frequency, given by:

$$
\omega_{p}=\frac{1}{\sqrt{L_{s} C_{P}}}
$$

and $Q_{p}$ is the loaded quality factor, given by:

$$
Q_{p}=\frac{R_{P}}{\omega_{p} L_{s}}=\omega_{p} C_{P} R_{P}
$$

The maximum voltage gain $\left(A_{v m}\right)$ can be found by taking the derivative of (13) with respect to $\omega$, substituting

$$
\begin{aligned}
& \frac{d}{d \omega}\left(A_{v}\right)=0 \text { and } \omega=\omega_{r} . \\
& \omega_{r}=\omega_{p} \sqrt{1-\frac{1}{2 Q_{p}^{2}}}
\end{aligned}
$$

where $\omega_{r}$ is the resonant frequency.

$$
A_{v m}=\frac{V_{o u t, r m s}}{V_{i n, r m s}}=\frac{Q_{p}}{\sqrt{1-\frac{1}{4 Q_{p}^{2}}}}
$$

For $Q_{p}>>1$, the (16) and (17) become,

$$
\begin{gathered}
\omega_{r} \cong \omega_{p} \\
A_{v m} \cong Q_{p}
\end{gathered}
$$

The equivalent parallel capacitance $C_{p}$ can be calculated by combining (14) and (18), resulting in the following equation:

$$
\begin{gathered}
\omega_{r} \cong \omega_{p} \\
C_{p}=\frac{1}{\omega_{r}^{2} L_{s}}
\end{gathered}
$$

By utilizing (20), at a given operating frequency, the values of $C_{p}$ and $L_{s}$ are extracted using DE. The equivalent parallel resistance $R_{p}$ can be calculated using (15) and (19), resulting in the following equation:

$$
R_{P}=A_{v m} \omega_{r} L_{s}
$$

The optimization process in DE is based on the minimization/maximization of an objective function. Therefore, a suitable objective function must be defined to begin the optimization process. Fig.5. shows the typical gain versus frequency characteristics of parallel loaded resonant circuit. It can be observed from Fig.5., a unique frequency $\left(\omega_{r}\right)$ exists at the maximum voltage. This unique frequency is treated as an objective function for the optimization problem, which in this case is minimization of (18).

$$
J=\omega_{r}-\omega_{p}
$$

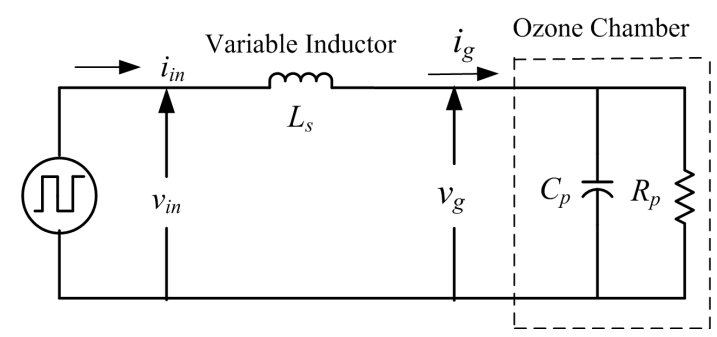

Fig.4. An equivalent circuit of ozone chamber and variable inductor.

Using (22) as the objective function, an optimization problem to determine $C_{p}, R_{p}$ and external inductance $L_{s}$ can be defined. The ozone chamber parameters can therefore be extracted by the following process: for a given resonant frequency (operating frequency), the DE method is applied to update the parameters by minimizing (22).

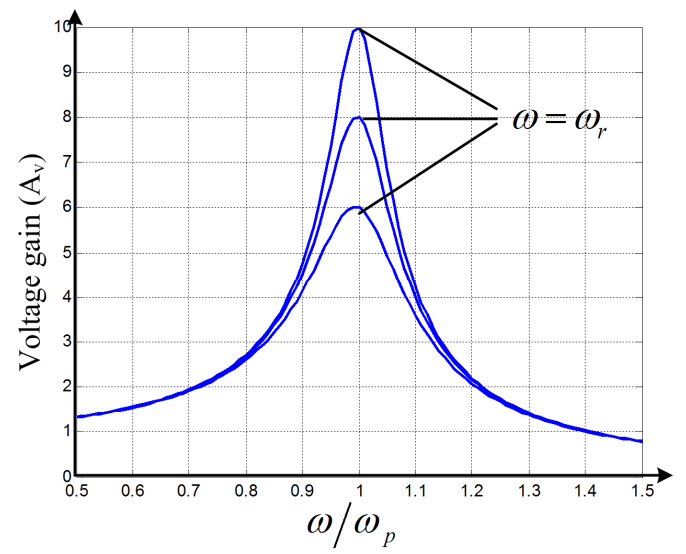

Fig.5. Frequency versus voltage gain of parallel loaded circuit.

\section{RESULTS FOR CHAMBER PARAMETER EXTRACTION USING DE}

The population size $(N P)$ is chosen to be 30 . This is a reasonable choice; typical values of $N P$ ranged between $5 D$ to $10 D$. The maximum generation number $\left(G_{\max }\right)$ is set to 100. The mutation scaling factor $(F)$ is set at 0.4 . The crossover rate $(C R)$ is chosen to be 0.5 . These values are adopted according to [18]. In chamber models, usually the 
capacitance is found to be in pico Farad, and most of the cases, less than $200 \mathrm{pF}$ [19]. However, parallel resistance $R_{p}$ value is very high [7]. In view of these data, the search ranges were set as follows: $R_{p} \in[300 \mathrm{k} \Omega, 650 \mathrm{k} \Omega], C_{p} \in$ $[120 \mathrm{pF}, 175 \mathrm{pF}], L_{s} \in[90 \mathrm{mH}, 340 \mathrm{mH}]$. In this work, the $\mathrm{DE} / \mathrm{best} / 1 / \exp$ strategy is used. In this nomenclature, the word "best" defines the best vector from the current population, "1" specifies number of difference vector and "exp" describes the exponential crossover technique. Due to stochastic nature of the DE, the model is executed for 10 times and the resulting average values are taken as the model parameters. The pseudo-code of the proposed modelling method is described by the following pseudocode:

\section{Step 1}

Selection of the control parameters of DE:

population size $N P$, crossover rate $C R$, mutation factor $F$ and the desired resonant frequency for the ozone chamber.

Step 2

Set the generation number $G=0$

and randomly initialize a population of $N P$ individuals with $X_{i, G}=\left[X_{1, i, G}, X_{2, i, G}, X_{3, i, G}, \ldots . ., X_{D, i, G}\right]$ and each individual uniformly distributed in the range $\left[X_{L}, X_{H}\right]$ as:

$X_{j, I, 0}=X_{L}+$ rand $[0,1]\left(X_{H}-X_{L}\right)$ where

$X_{L}=\left[X_{1, L}, X_{2, L}, \ldots, X_{D, L}\right]$ and

$X_{H}=\left[X_{1, H}, X_{2, H}, \ldots, X_{D, H}\right]$ with $i=[1,2, \ldots, N P]$.

Step 3

WHILE the stopping criterion is not satisfied

DO

FOR $\mathrm{i}=1$ to $N P$

\section{Step 3.1 Mutation Step}

Generate a donor vector $V_{i, G}=\left[V_{1, i, G}, V_{2, i, G}, \ldots \ldots ., V_{D, i, G}\right]$ corresponding to the $i_{t h}$ target vector $X_{i, G}$ using the differential mutation scheme of DE as:

$V_{i, G}=X_{r 1, G}+\mathrm{F}\left(X_{r 2, G}-X_{r 3, G}\right)$

\section{Step 3.2 Crossover Step}

Generate a trial vector $U_{i, G}=\left[U_{l, i, G}, U_{l, i, G}, \ldots \ldots ., U_{D, i, G}\right]$

for the $\mathrm{i}_{\text {th }}$ target vector $\mathrm{X}_{\mathrm{i}, \mathrm{G}}$ through the exponential

crossover

strategy.

\section{Step 3.2.1 Applying Boundary Constraints}

IF $U_{j, i, G}<X_{L}$, THEN $U_{j, i, G}=X_{L}-\operatorname{rand}[0,1]\left(X_{H}-X_{L}\right)$ ELSEIF $U_{j, i, G}>X_{H}$, THEN $U_{j, i, G}=X_{H}-\operatorname{rand}[0,1]\left(X_{H}-X_{L}\right)$ END IF

\section{Step 3.3 Selection and Evaluation Step}

Evaluate the trial vector $U_{i, G}$

IF $J\left(U_{i, G}\right)<J\left(X_{i, G}\right)$, THEN $X_{i, G+I}=U_{i, G}$

ELSE $X_{i, G+1}=X_{i, G}$.

END IF

END FOR

\section{Step 3.4 Increase the Generation Count}

$G=G+1$

END WHILE

The chamber parameters $\left(R_{p}\right.$ and $\left.C_{p}\right)$ are estimated at given operating frequencies. There is unique set of variable inductor $\left(L_{s}\right)$ and chamber parameter values for each given operating frequency. The extracted chamber parameter values are summarized in Table 1.
Table 1. Parameters of ozone chamber $\left(R_{p}\right.$ and $\left.C_{p}\right)$, estimated using the DE method.

\begin{tabular}{cccc}
\hline $\begin{array}{c}\text { Resonant } \\
\text { Frequency } \\
(\mathrm{kHz})\end{array}$ & $\begin{array}{c}\text { Variable } \\
\text { Inductance } \\
L_{\mathrm{s}}(\mathrm{mH})\end{array}$ & $\begin{array}{c}\text { Chamber } \\
\text { Capacitance } \\
C_{p}(\mathrm{pF})\end{array}$ & $\begin{array}{c}\text { Chamber } \\
\text { Resistance } \\
R_{p}(\mathrm{k} \Omega)\end{array}$ \\
\hline 24.3 & 289.6 & 148.99 & 576.76 \\
26.4 & 243.9 & 149.86 & 526.4 \\
27.1 & 229.7 & 151.55 & 507.4 \\
29.3 & 193.9 & 152.70 & 463.4 \\
31.5 & 168.7 & 153.40 & 432.0 \\
33.7 & 145.3 & 154.37 & 399.0 \\
35.5 & 128.5 & 158.00 & 371.0 \\
37.2 & 116.5 & 158.73 & 350.7 \\
40.8 & 96.3 & 160.58 & 322.0 \\
\hline
\end{tabular}

\section{EXPERIMENTAL VALIDATION}

\section{A. Power electronics set-up.}

The experimental setup to confirm the accuracy of the modelling method is shown in Fig.6.a). It consists of a high frequency half bridge PWM inverter, variable inductor, a gate drive circuit and an ozone chamber. The half bridge inverter converts DC voltage into high frequency square wave voltages. The IRFP460 MOSFET with freewheeling body diode is used as a switching device. The variable inductor is designed by the area product $\left(A_{p}\right)$ approach [20].

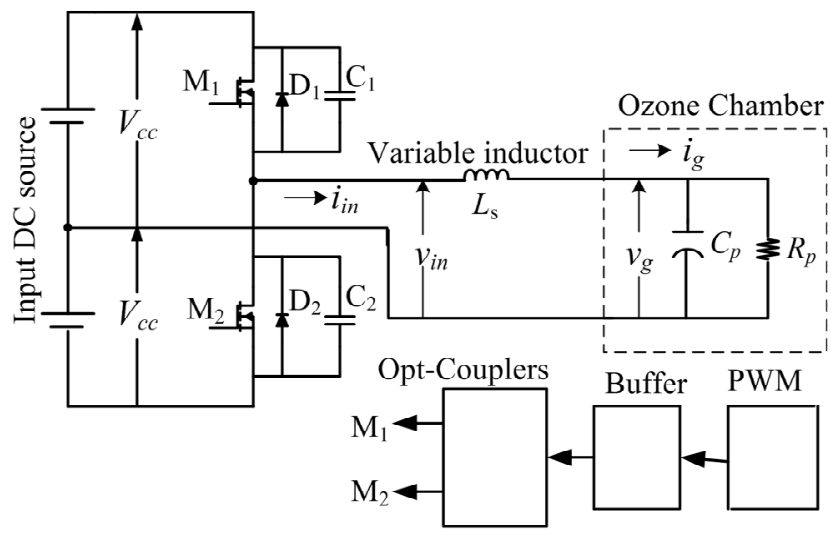

(a)

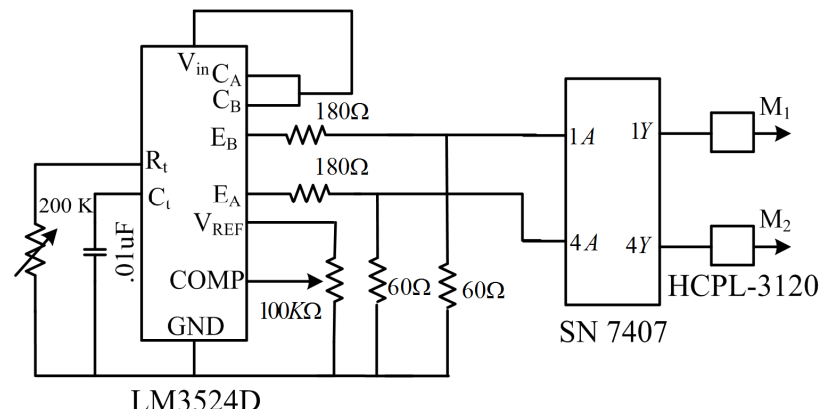

(b)

Fig.6. a) Experimental set up b) Gate drive circuit. 
The ETD-59, 3C90 ferrite core is used to build the variable inductor. The gating signals for MOSFETs $\mathrm{M}_{1}-\mathrm{M}_{4}$ are provided by the gate drive circuit as shown in Fig.6.b). The gate drive circuit provides stable $\mathrm{AC}$ sweep frequency up to $100 \mathrm{kHz}$. These signals are generated and regulated by the pulse width modulator (PWM) IC LM3524D. The frequency of voltage controlled oscillator (VCO) can be determined by the values of $R$ and $C$ connected at pins $R_{t}$ and $C_{t}$. The frequency of PWM output signals can be changed by varying the resistance connected with pin $R_{t}$. The duty cycle of the outputs can be varied by voltage applied at COMP pin of the PWM. The outputs of PWM are taken from NPN transistors, which are driven $180^{\circ}$ out of phase. The outputs of PWM are used to drive inputs of SN7407 buffer. The two outputs of buffer are used to drive the two HCPL-3120 optcouplers. The outputs of the opt-couplers provide the gating signal for half bridge inverter [21].

\section{B. Chamber construction.}

The ozone chamber consists of two parallel-electrodes, the top and bottom electrode are made from aluminium mesh and copper, respectively. The length of each electrode is $120 \mathrm{~mm}$ and its width is $70 \mathrm{~mm}$. Muscovite mica with $0.1 \mathrm{~mm}$ thickness is used as a dielectric to cover the bottom electrode. The dielectric prevents the arc discharge and limits the current flow. The discharge gap between electrodes is $1 \mathrm{~mm}$. The ozone chamber is supplied by normal air at flow rate of 1.0 litre per minute. Fig.7. shows the photograph of the complete experimental set-up.

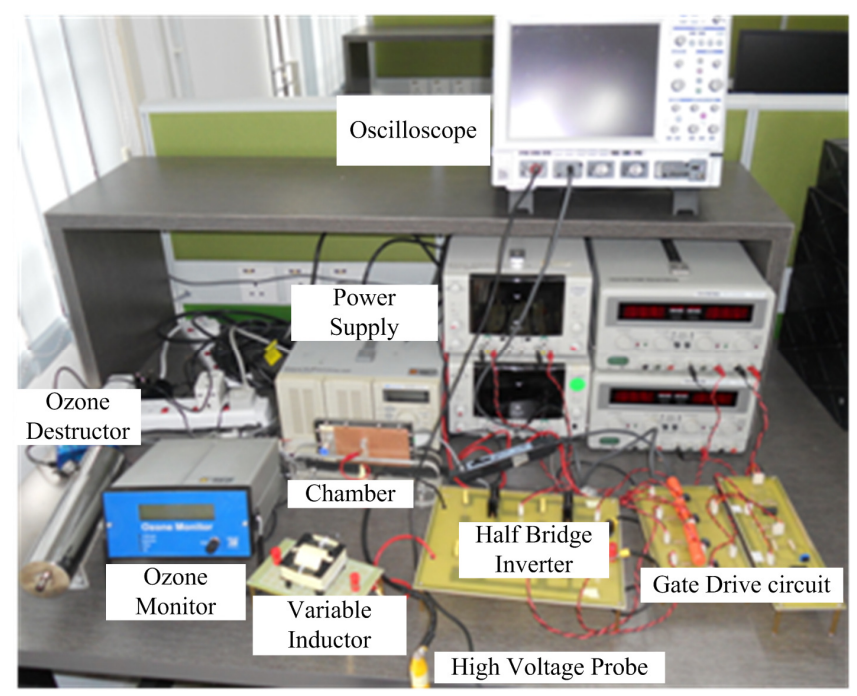

Fig.7. The photograph of experimental setup.

\section{Experimental procedure.}

(a) First, the value of the variable inductor $\left(L_{s}\right)$ is adjusted to be as close as possible to the value obtained by DE extraction (Table 1.). This is achieved by varying the air gap spacing between the cores.

(b) The switching frequency of inverter is varied slowly by varying $R_{t}$ of the gate drive circuit until resonance is achieved. (c) The value of gain at this resonant frequency is noted. Using Eqns. (20) and (21) the values of $C_{p}$ and $R_{p}$ are computed, respectively.

(d) The procedures (a)-(c) are repeated for another value $L_{s}$ as stated in Table 1 . Consequently, the chamber parameters in the frequency range of $24.3-40.8 \mathrm{kHz}$ are determined. They are shown in Table 2.

(e) For illustration, the voltage $\left(v_{g}\right)$ and current $\left(i_{g}\right)$ waveforms of the ozone chamber at three different resonant frequencies $(31.6,35.5$ and $37.2 \mathrm{kHz})$ are shown in Fig. 8 . The input voltage to the inverter is fixed at $35 \mathrm{~V}$. As can be seen, both the voltage and current waveforms are pure sinusoidal, which indicate that the circuit operates at resonance at all these frequencies. During the positive and negative cycles of current waveform, high frequency current spikes can be seen, which confirms the occurrence of discharges [7-9-19].

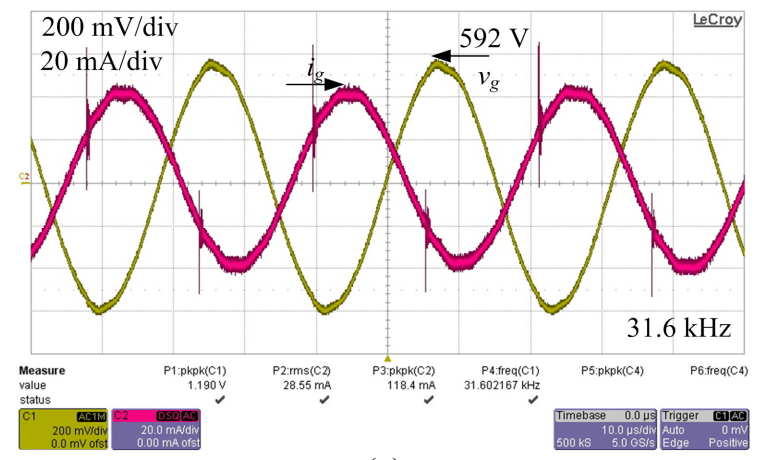

(a)

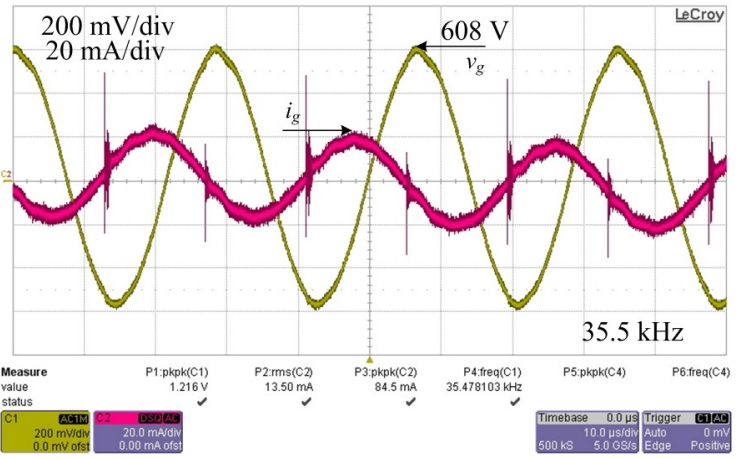

(b)

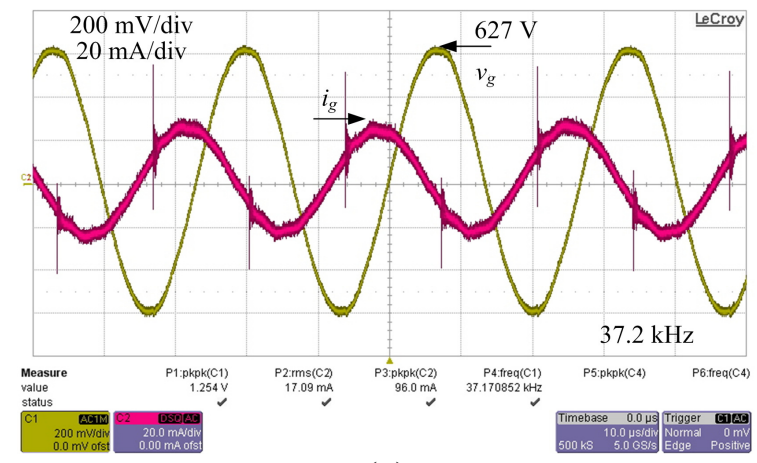

(c)

Fig.8. Voltage and current waveforms of the ozone chamber at various resonant frequencies. At resonant frequency of a) $31.6 \mathrm{kHz}$, b) $35.5 \mathrm{kHz}$, and c) $37.2 \mathrm{kHz}$. 
Table 2. Parameters of ozone chamber $\left(R_{p}\right.$ and $\left.C_{p}\right)$, obtained experimentally.

\begin{tabular}{ccccc}
\hline $\begin{array}{c}\text { Variable } \\
\text { Inductance } \\
L_{s}(\mathrm{mH})\end{array}$ & $\begin{array}{c}\text { Resonant } \\
\text { Frequency } \\
(\mathrm{kHz})\end{array}$ & $\begin{array}{c}\text { Voltage } \\
\text { Gain } \\
\left(A_{v m}\right)\end{array}$ & $\begin{array}{c}\text { Chamber } \\
\text { Capacitance } \\
C_{p}(\mathrm{mF})\end{array}$ & $\begin{array}{c}\text { Chamber } \\
\text { Resistance } \\
R_{p}(\mathrm{k} \Omega)\end{array}$ \\
\hline 290.6 & 24.3 & 12.58 & 147.6 & 558.2 \\
243.3 & 26.4 & 12.24 & 149.5 & 508.9 \\
230.0 & 27.1 & 12.65 & 150.7 & 496.1 \\
193.0 & 29.3 & 12.80 & 152.5 & 454.8 \\
168.0 & 31.5 & 13.00 & 153.0 & 432.3 \\
145.0 & 33.7 & 13.10 & 155.0 & 402.2 \\
128.0 & 35.5 & 13.26 & 157.0 & 377.5 \\
116.0 & 37.2 & 13.47 & 158.0 & 365.2 \\
95.0 & 40.8 & 13.68 & 161.5 & 333.2 \\
\hline
\end{tabular}

\section{Results and discussion.}

Fig.9.a) compares the extracted (using DE) and experimental values of $C_{p}$ with the variations of inverter switching frequency. As the frequency increases, the value of $L_{s}$ is decreased appropriately to maintain resonance. From the figure, it can be seen that $C_{p}$ increases slightly (less than $10 \%$ ) as the frequency is almost doubled. This is consistent with the observations made by other researchers, for example [22]. Fig.9.b) shows the comparison for $R_{p}$. With increasing frequency, $R_{p}$ decreases; this trend is consistent with results published in [19].

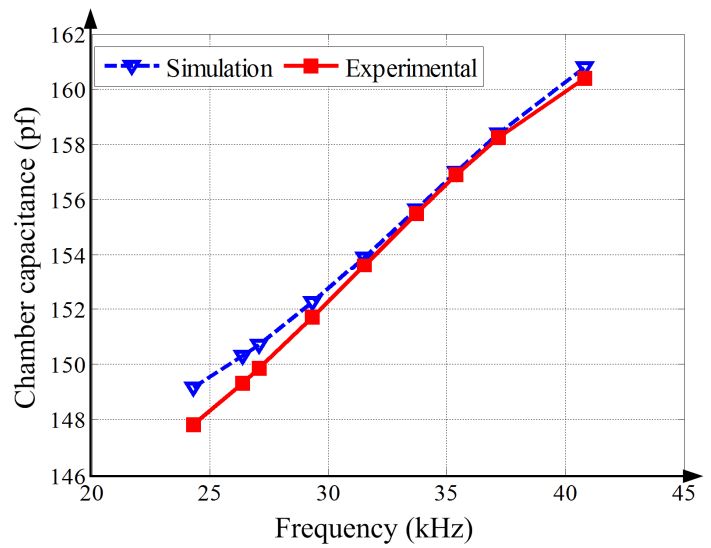

(a)

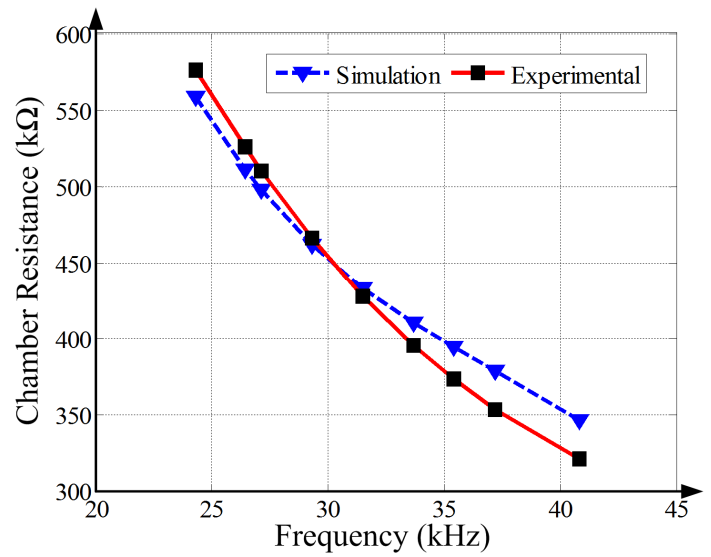

(b)

Fig.9. Comparison between the extracted and experimental results a) chamber capacitance b) chamber resistance.
The deviations in the extracted and experimental values of $C_{p}$ and $R_{p}$ may be due to the following reasons: (1) the variable inductor values used in the experiment are not the same as those obtained from extracted results. Experimentally, it is very difficult to build an inductor with an exact value as shown in Table 2. (2) Physically, the chamber capacitance is the combination of discharge gap $\left(C_{a}\right)$ and dielectric $\left(C_{g}\right)$ capacitance, respectively. However, in the linear model, they are lumped as $C_{p}$. During experiment, $C_{a}$ decreases due to breakdown of oxygen in the discharge gap, which causes a decrease in the experimental value of $C_{p}$. As can be observed from Tables 1 and 2, the experimental value of $C_{p}$ is always lower than the extracted value. The maximum deviation between the two is $0.93 \%$. Furthermore, as the voltage gain is also directly related to $C_{p}$, variation in the latter causes the voltage gain to vary. Since $R_{p}$ is calculated using this gain, it follows that the variation in $C_{p}$ will cause deviation in $R_{p}$. The maximum deviation between the extracted and experimental values of $R_{p}$ is $3.5 \%$.

\section{CONCLUSIONS}

In this paper, a new method to find ozone chamber parameters using DE has been proposed. This method is suitable to estimate the values of chamber parameters at high frequencies regardless of experimental constraints. The analysis and methodology to evaluate the ozone chamber parameters has been fully described. The proposed method can be used as a useful tool to design power supplies at various frequencies. Furthermore, it eliminates the dependence of Lissajous figure to obtain parameters. The accurateness of the proposed modelling method is validated with experimental results which are found to be in good agreement and confirm the validity of obtained parameters.

\section{ACKNOWLEDGMENT}

The authors would like to thank the Ministry of Education, Malaysia for funding the work under the Research University Grant (RUG) Scheme. The authors also thank the Universiti Teknologi Malaysia for managing the project under Vot. No. 2423.00G40.

\section{REFERENCES}

[1] Guzel-Seydim, Z.B., Greene, A.K., Seydim, A.C. (2004). Use of ozone in the food industry. Lebensmittel-Wissenschaft und-Technologie, 37 (4), 453-460.

[2] Kogelschatz, U. (2002). Filamentary, patterned, and diffuse barrier discharges. IEEE Transactions on Plasma Science, 30 (4), 1400-1408.

[3] Amjad, M., Salam, Z. (2014). Analysis, design, and implementation of multiple parallel ozone chambers for high flow rate. IEEE Transactions on Industrial Electronics, 61 (2), 753-765.

[4] Facta, M., Salam, Z., Buntat, Z. (2009). The development of ozone generation with low power consumption. In Innovative Technologies in Intelligent Systems and Industrial Applications, 25-26 July 2009. IEEE, 440-445. 
[5] Koudriavtsev, O., Shengpei, W., Konishi, Y., Nakaoka, M.A. (2002). Novel pulse-densitymodulated high-frequency inverter for silentdischarge-type ozonizer. IEEE Transactions on Industry Applications, 38 (2), 369-378.

[6] Oleg, K., Sergey, M., Nakaoka, M. (2002). Frequency characteristics analysis and switching power supply designing for dielectric barrier discharge type load. In VIII IEEE International Power Electronics Congress, 20-24 October 2002. IEEE, 222-227.

[7] Alonso, J.M., Valdés, M., Calleja, A.J., Ribas, J., Losada, J. (2003). High frequency testing and modeling of silent discharge ozone generators. Ozone: Science \& Engineering: The Journal of the International Ozone Association, 25 (5), 363-376.

[8] Ponce, M., Aguilar, J., Fernandez, J., Beutelspacher, E., Calderon, J.M., Cortes, C. (2004). Linear and non linear models for ozone generators. In 9th IEEE International Power Electronics Congress, 17-22 October 2004. IEEE, 251-256.

[9] Ponce-Silva, M., Aguilar-Ramirez, J., Beutelspacher, E., Calderon, J.M., Cortes, C. (2007). Single-switch power supply based on the class E shunt amplifier for ozone generators. In Power Electronics Specialists Conference, 17-21 June 2007. IEEE, 1380-1385.

[10] Haverkamp, R.G., Miller, B.B., Free, K.W. (2002). Ozone production in a high frequency dielectric barrier discharge generator. Ozone: Science \& Engineering: The Journal of the International Ozone Association, 24 (5), 321-328.

[11] Olivares, V.H., Ponce-Silva, M., Osorio, R., Juarez, M. (2007). DBD modeling as a function of waveforms slope. In Power Electronics Specialists Conference, 17-21 June 2007. IEEE, 1417-1422.

[12] Guofeng, J., Wei, Z., Zhengwei, Y., Zhiyong, H., Yuanjia, S., Dongdong, W., Gan, T. (2012). Image segmentation of thermal waving inspection based on particle swarm optimization fuzzy clustering algorithm. Measurement Science Review, 6 (12), 296301.

[13] Meo, A., Profumo, L., Rossi, A., Lanzetta, M. (2013). Optimum dataset size and search space for minimum zone roundness evaluation by genetic algorithm. Measurement Science Review, 13 (3), 100-107.
[14] Hasan, M., Reaz, M. (2012). Hardware prototyping of neural network based fetal electrocardiogram extraction. Measurement Science Review, 12 (2), 5255.

[15] Price, K.V., Storn, R.M., Lampinen, J.A. (2005). Differential Evolution : A Practical Approach to Global Optimization. Springer.

[16] Ishaque, K., Salam, Z., Taheri, H., Shamsudin, A. (2011). A critical evaluation of EA computational methods for Photovoltaic cell parameter extraction based on two diode model. Solar Energy, 85 (9), 17681779.

[17] Ordiz, C., Alonso, J.M.J., Costa, M.A.D., Ribas, J., Calleja, A.J. (2008). Development of a high-voltage closed-loop power supply for ozone generation. In Twenty-Third Annual IEEE Applied Power Electronics Conference and Exposition, 24-28 February 2008. IEEE, 1861-1867.

[18] Gamperle, R., Muller, S.D., Koumoutsakos, P. (2002). A parameter study for differential evolution. In WSEAS International Conference on Advances in Intelligent Systems, Fuzzy Systems, Evolutionary Computation. WSEAS, 293-298.

[19] Kinnares, V., Hothongkham, P. (2010). Circuit analysis and modeling of a phase-shifted pulsewidth modulation full-bridge-inverter-fed ozone generator with constant applied electrode voltage. IEEE Transactions on Power Electronics, 25 (7), 17391752.

[20] Mclyman, C.W.T. (1988). Transformer and Inductor Design Handbook. Third Edition. Marcel Dekker Publishing.

[21] Amjad, M., Salam, Z., Facta, M., Ishaque, K. (2012). A simple and effective method to estimate the model parameters of dielectric barrier discharge ozone chamber. IEEE Transactions on Instrumentation and Measurement, 61 (6), 1676-1683.

[22] Hothongkham, P., Kinnares, V. (2010). Measurement of an ozone generator using a phase-shifted PWM full bridge inverter. In International Power Electronics Conference, 21-24 June 2010. IEEE, 1552-1559.

Received July 12, 2013. Accepted April 10, 2014 